\title{
Estudo da qualidade das águas processadas em filtros de barro tradicionais contrapondo os filtros modernos
}

Caroline Vasconcelos Fernandes*(Graduanda em Engenharia Química na Universidade Federal de Campina Grande - UFCG)

Carla Gabriela Azevedo Misael (Graduanda em Engenharia Química na Universidade Federal de Campina Grande - UFCG);

Filipe J osé Ferreira Chaves (Graduando em Engenharia Química na Universidade Federal de Campina Grande - UFCG);

J osiele Souza Batista Santos (Graduanda em Engenharia Química na Universidade Federal de Campina Grande - UFCG);

Juan Nicolas Andrade Cavalcante (Graduando em Engenharia Química na Universidade Federal de Campina Grande - UFCG).

Suênia Fernandes de Vasconcelos (Graduanda em Engenharia Química na Universidade Federal de Campina Grande - UFCG);

*carolinefernandes.eq@gmail.com

\section{Resumo:}

A maior parte da água disponível na Terra está concentrada nos oceanos e mares, perfazendo 97,5\% do volume total. Entretanto, apenas $2,5 \%$ do volume total de água do planeta são doces, ressaltando que a maior parcela desta água $(68,9 \%)$, forma as calotas polares, as geleiras e neves eternas que cobrem os cumes das altas montanhas da Terra. Á vista disso, a preocupação da sociedade pela água sempre foi constante e desde cedo o homem vem na incessante procura por possibilidades de tornar a água potável ao seu alcance, pois nessa pequena parcela, existe ainda uma menor destinada ao consumo. Para tanto, sistemas de filtração foram desenvolvidos a fim de solucionar essa questão, desde o filtro de barro, o mais antigo, aos modernos aparelhos purificadores. Nesse contexto, este trabalho tem como objetivo avaliar a qualidade das águas processadas entre filtro de barro tradicional e o filtro purificador acoplado a torneira, através de análises físico-químicas, segundo o padrão da Portaria № 2.914, de 12 de Dezembro de 2011, do Ministério da Saúde. Através da alcalinidade e dureza da água verificou-se que os dois aparelhos de filtração são eficientes em manter a água dentro das especificações. Das análises do pH foi possível visualizar uma notável diferença entre as águas processadas no filtro de barro e filtro acoplado a torneira, inferindo que nem sempre os sistemas modernos de filtração são mais apropriados.

Pallavras-chave:

Filtração; qualidade; água potável 


\section{I ntrodução}

Todas as reações nos seres vivos necessitam de um veículo que as facilite e que sirva para regular a temperatura, em virtude do grande desprendimento de calorias resultante da oxidação da matéria orgânica. A água que é fundamental à vida, satisfaz completamente a estas exigências e se encontra presente em proporções elevadas na constituição de todos os seres vivos, inclusive no homem, onde atinge cerca de $75 \%$ de seu peso. Sua influência foi primordial na formação das aglomerações humanas (FUNASA, 2006).

A demanda de água potável vem aumentando assustadoramente com o crescimento populacional e o crescimento demográfico e econômico acelerado do Brasil, que nos últimos anos fez com que os recursos hídricos fossem demandados, em algumas regiões, além das disponibilidades. Além da escassez das reservas hídricas, tem sido intensificada a contaminação dos recursos d'água potável existentes, afetando assim, gravemente, a saúde humana (MMA, 2002). No ano de 2001, 2,4 bilhões de pessoas em todo o mundo já não viviam em condições aceitáveis de saneamento, enquanto 1,1 bilhões de pessoas não tinham sequer acesso a um adequado abastecimento de água (OPAS, 2001).

Desse período até os dias atuais, a necessidade de um aparelho que "purifique” a água está cada vez maior, pois o serviço de abastecimento público é precário e a manutenção do seu sistema de ductos faz com que a água que chega a residência dos brasileiros precise ser tratada. (BELLINGIERI, 2004).

No Brasil, a partir dos últimos anos do século XIX e início do século XX, começaram a surgir várias referências a aparelhos e equipamentos de filtragem e purificação de água para consumo doméstico. Destacaram-se nessa época os filtros de metal Berkfeld e Pasteur, como os mais indicados para a higienização domiciliar da água. Entretanto, estes aparelhos eram importados e utilizados por uma parcela muito pequena da população (BELLINGIERI, 2006). As técnicas para a produção de utensílios cerâmicos eram dominadas pelos imigrantes europeus e chegaram ao Brasil, a partir do final do século XIX. Algumas empresas brasileiras de cerâmica fundadas por imigrantes portugueses e italianos passaram a equipar as talhas que produziam os elementos filtrantes (velas), contribuindo para o surgimento da produção do filtro de água no Brasil. A década de 1930 pode ser considerada como início da generalização do consumo do filtro a vela e, a partir desse período, a evolução desse equipamento foi iniciada e novos processos de purificação foram desenvolvidos. Dessa maneira, os equipamentos acumulavam mais funções e além de eliminar partículas, também, esterilizava através do revestimento da prata coloidal (BELLINGIERI, 2004).

$\mathrm{O}$ filtro de barro é um dispositivo de gravidade, onde a água a ser filtrada passa através da vela e goteja do recipiente superior para o inferior do filtro, ficando ali armazenada para o consumo. $\mathrm{O}$ elemento filtrante utilizado, ou seja, a vela cerâmica, em filtros tipo gravidade, constitui-se numa peça de forma cilíndrica, oca, com paredes filtrantes em material cerâmico poroso, muitas vezes pode ser denominada de vela simples ou, ainda, vela tradicional (GUSMÃO, 2008). Segundo Bellingieri (2006), a função da vela é reter partículas e bactérias presentes na água, de forma que a água a ser consumida esteja isenta de poluição.

Com o passar das décadas, houve um declínio no uso dos filtros de barro em virtude do surgimento de produtos substitutos, com uma tecnologia mais avançada tais como os purificadores que usavam o mecanismo de retro lavagem, ou que possuem uma maior vazão. Estes filtros acoplados as torneiras são ligados à rede pública de abastecimento e, portanto, usam a pressão desta rede como uma forma de garantir a passagem da água por um elemento filtrante com porosidade mais fina, produzindo assim a retenção de particulados igualmente mais finos. Muitos destes equipamentos têm um elemento de carvão ativado capaz de eliminar o cloro residual livre adicionado na água para desinfecção, remover alguma concentração do flúor presente e reduzir a cor (desde que não produzida por metais) (COHESP, 2015).

Entretanto, a atual crise hídrica e elétrica trouxe de volta para alguns lares um item que já dominou as cozinhas em décadas passadas, mas que, a partir dos anos 90, começou a perder espaço no mercado e passou a ser considerado até mesmo "ultrapassado". As vendas cresceram 19\% nos últimos seis meses e com o aumento da procura, os fabricantes projetam um faturamento de R $\$ 30$ milhões em 2015, ante uma receita de R 26 milhões no ano passado. Entre as principais vantagens e diferenciais 
do filtro de barro, está o baixo custo de manutenção e a capacidade de refrescar a água de modo natural, sem gasto de energia elétrica. O tradicional filtro São João, o mais vendido até hoje pela Stéfani, recebeu a classificação P1 do Inmetro, a melhor concedida aos purificadores que retêm as menores partículas (ALVARENGA, 2015).

Diante desta perspectiva, este trabalho tem como objetivo comparar os resultados das águas processadas pelo aparelho de filtração moderno (acoplado a torneira), com aqueles obtidos utilizando o filtro de barro tradicional, a fim de avaliar a eficiência dos sistemas em uso sobre a qualidade das águas.

\section{Metodologia}

Coletou-se uma amostra de água, do município de Cuité, localizado na microrregião do Curimataú Ocidental paraibano, sem filtração. De imediato, parte da mesma foi reservada para ser analisada e a outra, submetida a dois processos diferentes de filtração.

\subsection{Processos de filtração}

Primeiramente, iniciou-se o primeiro processo: filtração por filtro de barro. A água foi filtrada ao passar por meio da vela cerâmica, acoplada à base do compartimento superior, para o compartimento inferior. A filtração sucedeu-se de forma lenta, gota a gota, e contínua, durante algum tempo, até o esvaziamento do compartimento superior do filtro. O primeiro processo de filtração está apresentado na Figura 1. Ao fim desse processo, a água filtrada ficou retida no compartimento inferior, em seguida, retirou-se três alíquotas para serem analisadas.

Figura 1 - Etapas do processo de filtração em filtros de barro tradicionais.

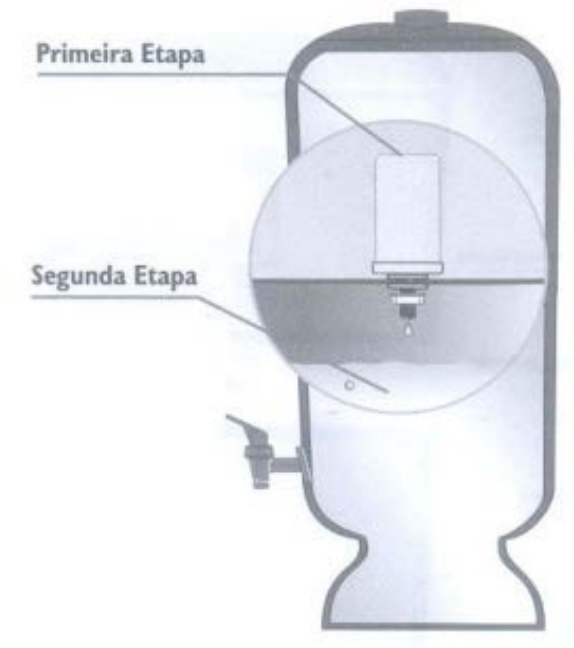

Fonte: Gusmão (2008).

Posteriormente, iniciou-se o segundo processo: filtração por filtro acoplado a torneira. Pelo fato de serem ligados à rede pública de abastecimento, usou-se a pressão dessa rede como uma forma de garantir a passagem da água por um elemento filtrante. O segundo processo de filtração está apresentado na Figura 2. Concluído esse passo, imediatamente coletaram-se três amostras para serem analisadas.

Figura 2 - Filtro acoplado a torneira. 


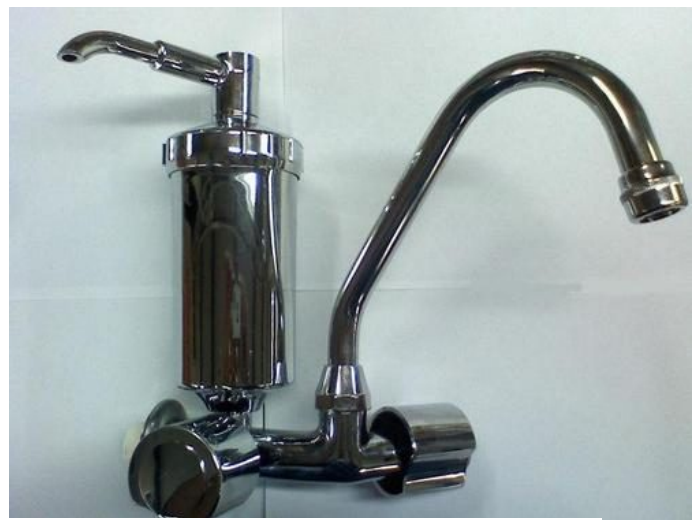

Fonte: http://adrenaline.uol.com.br/ (2015)

\subsection{Determinação da Alcalinidade}

Pipetou-se $25 \mathrm{~mL}$ da amostra de água sem filtrar, transferindo-a em seguida para um Erlenmeyer de $250 \mathrm{~mL}$. Após isso, adicionou-se duas gotas do indicador fenolftaleína e observou-se a cor. Em seguida, preencheu-se a bureta com solução de ácido sulfúrico $(0,02 \mathrm{~N})$ para titular a amostra. O primeiro volume de ácido sulfúrico gasto foi chamado de P. Ainda nessa amostra, adicionou-se duas gotas do indicador alaranjado de metila e observou-se a cor. Prosseguiu-se a titulação da mesma até a mudança de coloração. Logo após, anotou-se o volume de ácido sulfúrico consumido e chamou-o de T. O procedimento foi repetido em triplicata para o respectivo tipo de água, assim como para as demais.

\subsection{Determinação da Dureza}

Pipetou-se $25 \mathrm{~mL}$ da amostra de água sem filtrar e transferiu-se para um Erlenmeyer de $250 \mathrm{~mL}$. Em seguida, adicionou-se $3 \mathrm{~mL}$ de uma solução tampão $(\mathrm{pH}=10)$, duas gotas do indicador negro de eriocromo e observou-se a cor. Logo após, preencheu-se a bureta com uma solução padrão EDTA-Na $(0,025 \mathrm{~N})$ e titulou-se a amostra até a mudança de coloração. Imediatamente, anotou-se o volume de EDTA consumido. O procedimento foi repetido em triplicata para a respectiva amostra, assim como para as demais.

\subsection{Determinação do pH}

$\mathrm{O}$ pH das amostras foram determinados a partir de um pHmetro, conforme apresentado na Figura 3.

Figura 3 - pHmetro.

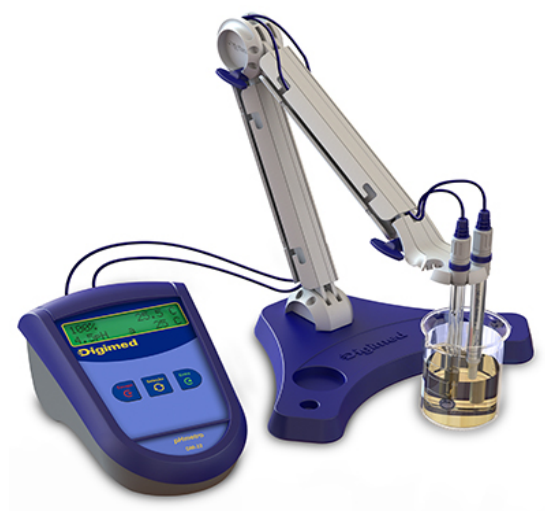

Fonte: Digimed (2015). 


\section{Resultados e discussão}

Levando em consideração as águas analisadas, denominou-se de água sem filtração, SF; água processada em filtro de barro tradicional, FB e água processada em filtro acoplado a torneira, FA.

\subsection{Alcalinidade da água}

A alcalinidade constitui-se em uma medida da capacidade da água de neutralizar os ácidos, servindo assim para expressar a capacidade de tamponamento da água e sua condição de resistir a mudanças do $\mathrm{pH}$ (MORAES, 2008). Os principais constituintes da alcalinidade são os bicarbonatos $\left(\mathrm{HCO}_{3}{ }^{-}\right)$, carbonatos $\left(\mathrm{CO}_{3}{ }^{-}\right)$e hidróxidos $\left(\mathrm{OH}^{-}\right)$, conforme a Tabela 1 apresenta.

Tabela 1 - Tabela para avaliar a alcalinidade da água.

\begin{tabular}{cccc}
\hline Resultados & \multicolumn{3}{c}{ Alcalinidades } \\
\hline $\mathbf{P}=\mathbf{0}$ & $\mathbf{O H}^{-}$ & $\mathbf{C O}^{{ }^{2-}}$ & $\mathbf{H C O}_{3}{ }^{-}$ \\
\hline $\mathbf{P}<\mathbf{T} / \mathbf{2}$ & Zero & Zero & $\mathrm{T}$ \\
\hline $\mathbf{P}=\mathbf{T} / \mathbf{2}$ & Zero & $2 \mathrm{P}$ & $\mathrm{T}-2 \mathrm{P}$ \\
$\mathbf{P}>\mathbf{T} / \mathbf{2}$ & Zero & $2 \mathrm{P}=\mathrm{T}$ & Zero \\
\hline $\mathbf{P}=\mathbf{T}$ & $2 \mathrm{P}-\mathrm{T}$ & $2(\mathrm{~T}-\mathrm{P})$ & Zero \\
\hline
\end{tabular}

P - Volume de ácido sulfúrico consumido após a adição da fenolftaleína.

$\mathrm{T}$ - Volume de ácido sulfúrico consumido após a adição do alaranjado de metila.

Fonte: Apostila Laboratório de Química Analítica - UFCG (2013)

Na determinação da alcalinidade, observou-se que nos três tipos de água, após a adição do indicador fenolftaleína, não houve mudanças de coloração, as mesmas permaneceram incolores, e, portanto, não houve necessidade de titular as amostras, assim, teve-se que $\mathrm{P}=0$. Prosseguindo a análise, após a adição do indicador alaranjado de metila, notou-se a coloração laranja, e com isso titulou-se as amostras com o ácido sulfúrico e o volume $\mathrm{T}$ consumido foi anotado. Os dados de alcalinidade são expressos como mg/L em $\mathrm{CaCO}_{3}$ por tratar-se de mistura de componentes. Os resultados obtidos para a respectiva análise dos três tipos de água, encontram-se na Tabela 2.

Tabela 2 - Resultados obtidos para análise da Alcalinidade

\begin{tabular}{lccccccc}
\hline & $\begin{array}{c}\text { Amostra 1 } \\
(\mathbf{m g} / \mathbf{L} \text { em } \\
\left.\mathbf{C a C O}_{3}\right)\end{array}$ & $\begin{array}{c}\text { Amostra 2 } \\
(\mathbf{m g} / \mathbf{L} \text { em } \\
\left.\mathbf{C a C O}_{3}\right)\end{array}$ & $\begin{array}{c}\text { Amostra 3 } \\
(\mathbf{m g} / \mathbf{L} \text { em } \\
\left.\mathbf{C a C O}_{3}\right)\end{array}$ & $\begin{array}{c}\text { P1,P2 } \\
\text { e P3 }\end{array}$ & $\begin{array}{c}\text { T1 } \\
(\mathbf{m L})\end{array}$ & $\begin{array}{c}\text { T2 } \\
(\mathbf{m L})\end{array}$ & $\begin{array}{c}\text { T3 } \\
(\mathbf{m L})\end{array}$ \\
\hline SF & 25,2 & 25,2 & 25,2 & 0 & 0,6 & 0,6 & 0,6 \\
FB & 58,8 & 58,8 & 58,8 & 0 & 1,4 & 1,4 & 1,4 \\
FA & 16,8 & 12,6 & 16,8 & 0 & 0,4 & 0,3 & 0,4 \\
\hline
\end{tabular}

P - Volume de ácido sulfúrico consumido após a adição da fenolftaleína.

T - Volume de ácido sulfúrico consumido após a adição do alaranjado de metila.

Fonte: Dados da pesquisa.

Segundo o padrão de potabilidade estabelecido pela legislação da portaria n ${ }^{0}$ A Portaria no 2.914, de 12 de dezembro de 2011 do Ministério da Saúde, não há valor mínimo ou máximo permitido para a alcalinidade da água, pois a mesma não representa risco potencial à saúde pública. Entretanto, a alcalinidade constitui-se em um parâmetro importante no controle de determinados processos unitários utilizados em estações de tratamento de águas para abastecimento. A partir da comparação da da Tabela 2 com a Tabela 1, notou-se que todas as águas apresentaram alcalinidade devido a bicarbonatos, o que geralmente ocorre em amostras com $\mathrm{pH}$ inferior a 8,3.

Segundo Chaoman e Kimstack (1992), águas de baixa alcalinidade (menor que $24 \mathrm{mg} / \mathrm{L}$ de $\mathrm{CaCO}_{3}$ ) apresentam baixa capacidade de tamponamento e, assim são susceptíveis às mudanças no 
pH. Assim, observou-se que a água do filtro acoplado (FA) apresentou as mais baixas alcalinidades; os motivos dessa discrepância em relação as outras devem ser diversos e de natureza desconhecida, que nesse caso precisaria de análises mais aprofundadas para saber os reais motivos dessa baixa alcalinidade. Já a água do filtro de barro (FB), apresentou-se acima do valor de $24 \mathrm{mg} / \mathrm{L} \mathrm{em} \mathrm{CaCO}_{3}$, ou seja, apresenta uma boa capacidade de tamponamento e não é apta a grandes variações no pH.

\subsection{Dureza da água}

A dureza da água é a propriedade relacionada com a concentração de íons de determinados minerais dissolvidos nesta substância. Esse fenômeno se deve à presença de determinados cátions na água, principalmente os cátions de cálcio e magnésio. Os dados de dureza da água são expressos como mg/L em $\mathrm{CaCO}_{3}$. A Tabela 3 apresenta a classificação das águas quanto a dureza.

Tabela 3 - Classificação das águas quanto ao grau de dureza

\begin{tabular}{cc}
\hline DUREZA TOTAL $\left(\mathbf{m g} / \mathrm{L} \mathrm{CaCO}_{3}\right)$ & CLASSIFICAÇÃO \\
\hline$<\mathbf{1 5}$ & Muito branda \\
de 15 a 50 & Branda \\
de 50 a 100 & Moderadamente branda \\
de 100 a 200 & Dura \\
$>\mathbf{2 0 0}$ & Muito dura \\
\hline
\end{tabular}

Fonte: Apostila Laboratório de Química Analítica - UFCG.

Os resultados obtidos para a análise da Dureza da água sem filtração e das águas processadas em filtro de barro tradicional e o moderno acoplado a torneira, encontram-se na Tabela 4.

Tabela 4 - Resultados obtidos para análise da Dureza da água

\begin{tabular}{|c|c|c|c|c|c|c|c|}
\hline & $\begin{array}{c}\text { Amostra } \\
1 \\
(\mathrm{mg} / \mathrm{L} \\
\left.\mathrm{CaCO}_{3}\right)\end{array}$ & $\begin{array}{c}\text { Amostra } \\
2 \\
(\mathrm{mg} / \mathrm{L} \\
\left.\mathrm{CaCO}_{3}\right)\end{array}$ & $\begin{array}{c}\text { Amostra } \\
3 \\
(\mathrm{mg} / \mathrm{L} \\
\left.\mathrm{CaCO}_{3}\right)\end{array}$ & Classificação & $\begin{array}{c}\text { V1 (mL } \\
\text { de } \\
\text { EDTA) }\end{array}$ & $\begin{array}{c}\text { V2 (mL } \\
\text { de } \\
\text { EDTA) }\end{array}$ & $\begin{array}{c}\text { V3 (mL } \\
\text { de } \\
\text { EDTA) }\end{array}$ \\
\hline SF & 50 & 40 & 50 & BRANDA & 1 & 0,8 & 1 \\
\hline FB & 45 & 45 & 45 & BRANDA & 0,9 & 0,9 & 0,9 \\
\hline FA & 30 & 40 & 40 & BRANDA & 0,6 & 0,8 & 0,8 \\
\hline
\end{tabular}

V1, V2 e V3 - Volumes de EDTA consumidos durante a titulação.

Fonte: Dados da pesquisa.

A Portaria $\mathrm{n}^{\circ}$ 2.914, de 12 de dezembro de 2011, limita a dureza em $500 \mathrm{mg} / \mathrm{L} \mathrm{CaCO}_{3}$ como padrão de potabilidade. Diante disso, verificou-se que todas as águas (SF, FB e FA) apresentaram a classificação do tipo "BRANDA", em conformidade com a Tabela 3, e se enquadram no padrão de potabilidade exigido pela Lei. A partir dessa análise percebeu-se que o sistema de filtração da água, seja no FB ou FA, ou mesmo se não houver filtração, isso pouco influencia na dureza da água.

\subsection{Análise do pH}

$\mathrm{O}$ valor de $\mathrm{pH}$ é também um resultado importante para a composição dos chamados índices de qualidade de águas (IQA). A Portaria $\mathrm{n}^{\circ}$ 2.914, de 12 de dezembro de 2011 do Ministério da Saúde, recomenda que o $\mathrm{pH}$ da água seja mantido na faixa de 6,0 a 9,5. Os resultados obtidos para a análise do $\mathrm{pH}$ para os três tipos de água, podem ser observados na Tabela 5.

Tabela 5 - Resultados obtidos para análise do pH

\begin{tabular}{|c|c|c|c|}
\hline $\begin{array}{c}\text { ANÁLISE DE } \\
\text { PH }\end{array}$ & Amostra 1 & Amostra 2 & Amostra 3 \\
\hline
\end{tabular}




\begin{tabular}{|l|l|l|l|l|l|l|l|l|l|}
\hline SF & 4,03 & 4,31 & 4,21 & 4,13 & 4,08 & 4,00 & 4,36 & 4,24 & 4,14 \\
\hline FB & 8,09 & 7,90 & 8,11 & 8,14 & 7,09 & 7,27 & 7,84 & 7,90 & 7,97 \\
\hline FA & 4,37 & 4,25 & 4,29 & 4,29 & 4,35 & 4,40 & 4,52 & 4,42 & 4,37 \\
\hline
\end{tabular}

Fonte: Dados da pesquisa.

Através dos resultados adquiridos na Tabela 5, notou-se que a água do tipo SF apresentou baixo pH com uma média de 4,17. É importante salientar que água sem o processo de filtração é desaconselhável, uma vez que o excesso de produtos químicos, oriundos das estações de tratamento, pode tornar o consumo dessa água um tanto inviável.

A água do filtro acoplado (FA) exibiu média de 4,36, média essa que chama atenção, pois mesmo sendo sujeita a filtração, ainda assim apresentou pHs abaixo dos valores limites exigidos pela legislação. Em contrapartida, o filtro de barro (FB) apresentou melhor desempenho, uma vez que atendeu as exigências da Lei, exibindo uma média de $\mathrm{pH}=7,81$, que se encontra dentro da faixa estabelecida. Um dos fatores que determinam o pH da água é o gás carbônico dissolvido e a alcalinidade total. Assim, entende-se que a baixa alcalinidade apresentada pelas águas do FA, e elevada alcalinidade apresentada pelas águas do FB, conforme mostra a Tabela 2, pode ter exercido influência no $\mathrm{pH}$ das mesmas

Além dos resultados analisados, estudos publicados no livro The Drinking Water Book de Colin Ingram (2006) apontam que o filtro tradicional de barro, com câmara de filtragem de cerâmica microporosa é bastante eficiente na retenção de cloro, pesticidas, ferro, alumínio, chumbo e parasitas causadores de diarreias e dor abdominal. Nessa situação e diante dos dados obtidos o uso de água processada pelo filtro de barro tradicional não ocasionaria danos a saúde do indivíduo, diferentemente das outras águas analisadas. Nesse caso, a tradição do FB se sobrepõe a modernidade do FA.

\section{Conclusão}

Tendo em vista as informações discutidas e a análise dos resultados adquiridos, pode-se assegurar que existe a necessidade da água que se destina ao consumo humano, ser submetida a processos de filtração, ainda que a mesma tenha sido sujeita a vários procedimentos prévios nas estações de tratamento. Através da análise do $\mathrm{pH}$, fica evidente que o filtro de barro tradicional tem um melhor desempenho para processar uma água de melhor qualidade, uma vez que seu sistema de filtração permite que uma água, antes ácida, obtenha um pH indicado pela Lei. Além disso, em virtude dos problemas relacionados à potabilidade da água, as pessoas têm dado preferência ao consumo da água mineral, todavia, a retomada do filtro de barro além de processar uma água de maior qualidade, pode ser uma forma de diminuir os custos.

\section{Agradecimento}

Os autores desse trabalho agradecem a Universidade Federal de Campina Grande e a Unidade Acadêmica de Engenharia Química.

\section{Quality evaluation of processed water in traditional clay filters contrasting modern filters}

Albstract: The most of the water available on earth is concentrated in the oceans and seas, totaling $97,5 \%$ of the total volume. Only $2,5 \%$ of the total volume on planet water are sweets, highlighting the largest slice of this water $(68,9 \%)$, form the polar ice caps, glaciers and permanent snow that 
cover the tops of the highest Earth mountains. To the light of this, the concern of society by water has been always constant and early the man is in constant search for possibilities to make potable water at his fingertips, because this small portion, still exist a smaller for consumption. To this end, filtration systems have been developed to solve this issue, since the clay filter, the oldest, until the modern purifiers appliances. In this context, this work aims to evaluate the quality of processed water between traditional clay filter and the purifier filter coupled to tap, through physical and chemical analysis according to the standard of Ordinance no 2.914, de 12 de December of 2011, of the Healthy Ministry. Through the alkalinity and hardness of water was found that the two filtration devices are effective to maintain the water within the specifications. The $\mathrm{pH}$ analysis was possible to see a considerable difference between the processed water into the clay filter and the other filter inferring that not always the filtration modern systems are more appropriate.

Keywords: Filtration; quality; potable water

\section{Referências bibliográficas}

ADRENALINE -Como escolher um purificador de água. Disponível em: < http://adrenaline.uol.com.br/>. Acessado em 24 de setembro de 2015

ALVARENGA, D. Crise hídrica faz crescer procura por tradicionais filtros de barro. Disponível em: < http:/g1.globo.com/economia/crise-da-agua/noticia/2015/04/crise-hidrica-faz-crescer-procurapor-tradicionais-filtros-de-barro.html>. Acessado em: 10 de setembro de 2015.

BELLINGIERI, J. C. Água de beber: a filtração doméstica e a difusão do filtro de água. Anais do Museu Paulista, junho-dezembro, ano/vol. 12, nº 012, Universidade de ão Paulo - USP/2004. p. 161192.

BELLINGIERI, J.C. Uma análise da indústria de filtros de água no Brasil. In: $50^{\circ}$ CONGRESSO BRASILEIRO DE CERÂMICA, Blumenau. SC. v. 11, nº 3, maio-junho. p. 31-35, 2006.

BRASIL. FUNDAÇÃO NACIONAL DE SAÚDE. Manual prático de análise de água. Rev. Brasília: Fundação Nacional de Saúde, 2004.

BRASIL. MINISTÉRIO DA SAÚDE. SECRETARIA DE VIGILÂNCIA EM SAÚDE. COORDENAÇÃo GERAL DE VIGILÂNCIA EM SAÚdE AMBIENTAL. Portaria $\mathbf{n}^{\mathbf{0}}$ - $\mathbf{2 . 9 1 4}$. Brasília: Editora do Ministério da Saúde, 2011.

CHAPMAN, D; KIMSTACK, V. The selection of water quality variables The selection of water quality variables . London: Chapman \& Hall Ltd., 1992. p. 51-117.

CONTROLE HÍDRICO DE SÃO PAULO. Ph ideal para consumo humano. Disponível em: < http://cohesp.com.br/qual-ph-ideal-da-agua-para-consumo-humano/>. Acessado em: 25 de setembro de 2015.

DIGIMED - INSTRUMENTAÇÃO ANALÍTICA. PHmetro de Laboratório DM-22. Disponível

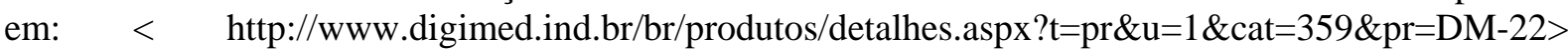
Acessado em 24 de Setembro de 2015.

GUSMÃO, P.T.R.de. Manual de Orientações - Filtro Doméstico. Proveniente da Pesquisa: "Filtros domésticos: Avaliação de sua eficácia e eficiência na redução de agentes patogênicos". Departamento de Engenharia Civil da Universidade Federal de Pernambuco. Recife, 2008.

INGRAM, C. The drinking water book. $2^{\mathrm{a}}$ ed. 2006 
MINISTÉRIO DA SAÚDE, “Portaria 36: Padrões de Potabilidade”. 1990.

MINISTÉRIO DO MEIO AMBIENTE DO BRASIL (MMA), Avaliação das águas do Brasil. Brasília, 2002.

MORAES, B.P. Curso Superior de Tecnologia em Saneamento Ambiental CESET/UNICAMP. Disponível em: < http://webensino.unicamp.br/disciplinas/ST502293205/apoio/2/Resumo_caracteriza_o_de_efluentes_continua_o.pdf $>$. Acessado em: 24 de setembro de 2015

ORGANIZAÇÃO PAN-AMERICANA DA SAÚDE, Água e Saúde. Disponível em: < https://www2.mppa.mp.br/sistemas/gcsubsites/upload/41/Agua\%20e\%20Saude\%20-

\%20Organizacao\%20Pan-Americana\%20da\%20Saude.pdf > Acessado em: 24 de setembro de 2015. 